\title{
Operators of Finite Rank in Unitary Representations of Exponential Lie Groups
}

\section{Detlev Poguntke}

Fakultät für Mathematik der Universität, Postfach 8640, D-4800 Bielefeld, Federal Republic of Germany

Let $G$ be a locally compact group and let $\pi$ be an irreducible unitary representation of $G$ (by unitary representation we always mean a strongly continuous unitary representation) such that the image of the $C^{*}$-hull $C^{*}(G)$ of $L^{1}(G)$ under $\pi$ contains the compact operators. Then $\pi\left(L^{1}(G)\right)$ "almost never" contains all compact operators but it is natural to ask whether it contains at least one nontrivial compact operator or, even better, operator of finite rank (and, consequently, a rank one projection). In this generality the answer is negative as was shown by Guichardet, pp. 65/66 in [9]. But one may restrict the questions to "nice" classes of type $I$ groups (where the assumption made on $\pi$ is automatically satisfied). Up to my knowledge, for connected Lie groups of type $I$ no counterexample is known. To the contrary, there are positive results. Dixmier has proved in $[8]$ that $\pi\left(L^{1}(G)\right)$ contains finite rank operators for all irreducible unitary representations $\pi$ of a nilpotent Lie group $G$. The same is true for semisimple Lie groups. Moreover, for solvable Lie groups $G$ Charbonnel has shown, [7], that $\pi\left(L^{1}(G)\right.$ ) (even $\pi(\mathscr{D}(G)$ ) contains (generalized) trace class operators for all normal factor representations $\pi$ of $G$ which implies that $\pi\left(L^{1}(G)\right)$ contains compact operators for all irreducible unitary representations $\pi$ of a solvable Lie group $G$ of type $I$. It is the purpose of this article to add another positive result, namely to prove the following theorem.

Theorem. Let $G$ be an exponential Lie group and let $\pi$ be an irreducible unitary representation of $G$. Then $\pi\left(L^{1}(G)\right)$ contains a projection of rank one.

The paper consists of two parts. In the first part we introduce some notations and prove three lemmata which are of some independent interest. In the second part we give the proof of the theorem. Meanwhile, this theorem was also proved by Ludwig in his unpublished manuscript "Operators with smooth kernels and irreducible representations of a solvable Lie group". Ludwig's construction is more explicit than mine what may have some advantages. At a crucial point of my proof operators of finite rank are constructed by using the symmetry of a certain algebra. I will shortly introduce the concept of symmetry and explain the relation to finite rank operators. In fact, I got interested in the existence of finite rank operators by asking for the symmetry of group algebras of exponential Lie 
groups, and in some sense the present article may be considered as an appendix or continuation of my paper [15].

Recall that an involutive Banach algebra $A$ is called symmetric if elements of the form $a^{*} a, a \in A$, have a real non-negative spectrum. This is, by the theorem of Shirali and Ford, equivalent to the fact that hermitean elements in $A$ have a real spectrum. Another useful equivalent characterization is the following, see e.g. [11]: For every simple (left) $A$-module $E$ there exists an irreducible involutive representation $\pi$ of $A$ in an Hilbert space $\mathscr{H}$ and an $A$-linear embedding $E \rightarrow \mathscr{H}$. From this characterization one deduces very easily (see also p. 311 in [13]) the following: If $a$ is an hermitean element in the symmetric algebra $A$ and $\lambda$ is a nonzero (real) number in the spectrum of $a$ then there exists an irreducible involutive representation $\pi$ of $A$ in the Hilbert space $\mathscr{H}$ and a non-zero $\xi$ in $\mathscr{H}$ with $\pi(a) \xi$ $=\lambda \xi$. Moreover, (closed involutive) subalgebras and quotients (modulo closed involutive ideals) of symmetric algebras are again symmetric. Concerning finite rank operators there is the following lemma which was already shown in [15], but for the convenience of the reader we repete its short proof.

Lemma 1. Let $A$ be a symmetric involutive Banach algebra. Suppose that $A$ has (up to unitary equivalence) precisely one irreducible involutive representation (by compact operators), say $\pi$. Then $\pi(A)$ contains a projection of rank one.

Proof. Let $a$ be any positive element (i.e. $\left.a=b^{*} b, b \in A\right)$ in $A$ with $\pi(a) \neq 0$. From the symmetry of $A$ and the uniqueness of $\pi$ it follows that the spectrum of $a$ in $A$ coincides with the spectrum of the operator $\pi(a)$. Let $\lambda$ be the largest eigenvalue of the positive compact operator $\pi(a)$, and let $\Gamma$ be a small positively oriented circle in the complex plane around $\lambda$ such that there is no spectral value of $\pi(a)$ on $\Gamma$ or in the interior of $\Gamma$ except $\lambda$. We adjoin an identity to $A, A^{\prime}:=A \oplus \mathbb{C}$, and form the integral

$$
b:=\frac{-1}{2 \pi i} \int_{\Gamma}(a-z)^{-1} d z \text { in } A^{\prime} .
$$

It is easy to see that $b$ lies in $A$ and that

$$
\pi(b)=\frac{-1}{2 \pi i} \int_{\Gamma}(\pi(a)-z)^{-1} d z
$$

is the projection on the $\lambda$-eigensapce $\mathscr{H}_{\lambda}$ of $\pi(a)$. The algebra $\pi(b A b)$ acts irreducibly on the finite-dimensional space $\mathscr{C}_{\lambda}$. From Burnside's theorem it follows that $\pi(b A b)$ contains projections of rank one.

We note (omitting the simple proof) that there is also partial converse of Lemma 1 in the following sense.

Remark. Let $A$ be a simple involutive Banach algebra having precisely one irreducible involutive representation $\pi$. If $\pi(A)$ contains nonzero operators of finite rank then $A$ is symmetric.

Moreover, the existence of finite rank operators in involutive representations of group algebras has consequences for the symmetry (or better: nonsymmetry) of these algebras, see the remark at the end of this article.

In the proof of the theorem $I$ will also use the concept of $*$-regularity (see $[4$, 3]). Let $A$ be an involutive Banach algebra, and let $C^{*}(A)$ be the $C^{*}$-hull of $A$. By 
$\operatorname{Priv}_{*}(A)$ (=*-primitive ideals) we denote the space of kernels of irreducible involutive representations of $A$, equipped with the hull-kernel topology. $A$ is called *-regular if the canonical map $\operatorname{Priv}_{*}\left(C^{*}(A)\right) \rightarrow \operatorname{Priv}_{*}(A)$ is an homeomorphism. A useful equivalent characterization of *-regularity is the following, see [4]: If $\sigma$ and $\tau$ are involutive representations of $A$ and if $\sigma^{\prime}$ and $\tau^{\prime}$ are the corresponding representations of $C^{*}(A)$ then $\operatorname{ker} \sigma \leqq \operatorname{ker} \tau$ implies $\operatorname{ker} \sigma^{\prime} \leqq \operatorname{ker} \tau^{\prime}$. From this characterization one obtains: If $I$ is a closed involutive ideal in the *-regular algebra $A$ then $I$ and $A / I$ are *-regular, too. It is known, [4], that group algebras of groups with polynomially growing Haar measure are *-regular; in particular, this applies to group algebras of nilpotent Lie groups.

Let $G$ be a locally compact group, and let $A$ be an involutive Banach algebra. Then we call $A$ an involutive $G$-algebra if there is given an homomorphic action $G \times A \rightarrow A,(x, a) \rightarrow a^{x}$, of $G$ on $A$ by isometric involutive isomorphisms such that $x \rightarrow a^{x}$ is a continuous function from $G$ in $A$ for each $a \in A$. If $A$ is an involutive $G$-algebra then $G$ acts (from the left) on the representations of $A$ by $(x \pi)(a):=\pi\left(a^{x}\right)$ and on $\operatorname{Priv}_{*}(A)$ by $x P:=P^{x^{-1}}$; in particular, if $P$ is the kernel of the irreducible involutive representation $\pi$, then $\operatorname{ker} x \pi=x P$. The following lemma may be considered as a more subtle (and more technical) version of Lemma 1 ; it will be used to construct a certain symmetric algebra.

Lemma 2. Let $G$ be a locally compact group, and let $A$ be an involutive G-algebra. Suppose further that $A$ is semisimple, *-regular and symmetric, that $\pi(A)$ consists of compact operators for all irreducible involutive representations $\pi$ of $A$, that $X:=\operatorname{Priv}_{*}(A)$ is a locally compact Hausdorff space, that $X$ is a transitive $G$-space and that the canonical mapping $G / G_{P} \rightarrow X$ is an homeomorphism for every $P \in X$ where $G_{P}$ is the stabilizer of $P$ in $G$. For every $a \in A$ the "Fourier transform" $\hat{a}: X \rightarrow \bigcup_{P \in X} A / P$ is defined by $\hat{a}(P):=a+P \in A / P$. Then there exist positive elements $p, q \in A$ with $p q=q p=p \neq 0$ such that $\hat{g}$ has an (arbitrarily small) compact support and that $\pi(q)$ is zero or an operator of rank one for all irreducible involutive representations $\pi$ of $A$.

Proof. First, we claim that if $\sigma$ and $\tau$ are irreducible involutive representations of $A$ with $\operatorname{ker} \sigma \cong \operatorname{ker} \tau$ then $\sigma$ and $\tau$ are equivalent. In particular, an irreducible representation is determined by its kernel. Since $A$ is *-regular the kernel of $\sigma^{\prime}$ is contained in the kernel of $\tau^{\prime}$ if $\sigma^{\prime}$ and $\tau^{\prime}$ denote the extensions of $\sigma$ and $\tau$ to the $C^{*}$ hull $C^{*}(A)$ of $A$, respectively. Hence $\tau^{\prime}$ may be considered as a representation of $C^{*}(A) / \operatorname{ker} \sigma^{\prime}$ which is isomorphic to the algebra of compact operators. Since the latter algebra has precisely one irreducible representation it follows that $\tau^{\prime}$ is equivalent to $\sigma^{\prime}$, and hence $\tau$ is equivalent to $\sigma$.

Let a point $P_{0}$ in $X$ and a (relatively compact) neighborhood $U$ of $P_{0}$ be given. We will construct an $q$ in $A$ with the indicated properties and with $\hat{q}\left(P_{0}\right) \neq 0, \hat{q}=0$ outside $U$. Let $\pi_{0}$ be a fixed irreducible unitary representation of $A$ with $\operatorname{ker} \pi_{0}$ $=P_{0}$, and let $\mathscr{H}$ be the representation space of $\pi_{0}$. The quotient algebra $A / P_{0}$ satisfies the assumptions of Lemma 1; it is symmetric as a quotient of $A$ and it has precisely one irreducible representation by the remark above. Hence we find an $a \in A$ such that $\pi_{0}(a)$ is a projection of rank one, and we may assume that $a$ is positive. 
The function $x \rightarrow \pi_{0}\left(a^{x}\right)$ from $G$ into the positive compact operators on $\mathscr{H}$ is continuous. Hence for every $\varepsilon>0$ there exists a compact neighborhood $W$ of the identity such that

$$
\mathrm{Sp}\left(\pi_{0}\left(a^{x}\right)\right) \leqq\{t \in \mathbb{R} ; 0 \leqq t \leqq \varepsilon \text { or }|1-t| \leqq \varepsilon\}
$$

and

$$
\left\|\pi_{0}\left(a^{x}\right)-\pi_{0}(a)\right\| \leqq \varepsilon \text { for all } x \in W .
$$

From these data one deduces very easily that for $\varepsilon<1 / 4$ (which we assume from now) and $x \in W$ the operator $\pi_{0}\left(a^{x}\right)$ has precisely one spectral (eigen) value in the circle $|z-1| \leqq 1 / 2$ in the complex plane and that this eigenvalue has multiplicity one.

Let $V:=W P_{0}, V$ is a compact neighborhood of $P_{0}$ in $X$. Let $K$ be the kernel of $V$, i.e. $K=k(V)=\bigcap_{P \in V} P$, let $A_{V}:=A / K$ be the quotient algebra and let $\varphi: A \rightarrow A_{V}$ be the quotient map. The space $\operatorname{Priv}_{*}\left(A_{V}\right)$ is homeomorphic to $V$ and every irreducible involutive representation of $A_{V}$ is equivalent to one of the form $x \pi_{0}$, $x \in W$. From the symmetry of $A_{V}$ it follows that the spectrum of $\varphi(a)$ in $A_{V}$ is contained in $\bigcup_{x \in W} \operatorname{Sp}\left(x \pi_{0}(a)\right)$ and hence in the union of the intervalls $[0, \varepsilon]$ and $[1-\varepsilon, 1+\varepsilon]$. Now we argue as in the proof of Lemma 1 . We adjoin a unit element to $A_{V}, A_{V}^{\prime}:=A_{V} \oplus \mathbb{C} 1$. Let

$$
\Gamma(t):=1+\frac{1}{2} e^{i t}, \quad 0 \leqq t \leqq 2 \pi,
$$

and let

$$
b:=\frac{-1}{2 \pi i} \int_{\Gamma}(\varphi(a)-z)^{-1} d z \in A_{V}^{\prime} .
$$

Then $b$ is contained in $A_{V}$ and, for $x \in W$,

$$
\left(x \pi_{0}\right)(b)=-\frac{1}{2 \pi i} \int_{\Gamma}\left(\pi_{0}\left(a^{x}\right)-z\right)^{-1} d z
$$

is the projection onto the (one dimensional) eigenspace of the unique eigenvalue of $\pi_{0}\left(a^{x}\right)$ inside $\Gamma$. Let $c$ be any element in $A$ with $\varphi(c)=b$. We choose an open neighborhood $Y$ of $P_{0}$, contained in $U \cap V$, and form the ideal $I:=k(X \backslash Y)$. Since $\pi_{0 \mid}$ is not zero and hence irreducible there exists an $d \in I$ with $\pi_{0}(d c) \neq 0$. The element $f:=(d c)^{*} d c=c^{*} d^{*} d c \in A$ has the following properties:

(1) $\hat{f}=0$ outside $Y$,

(2) $\pi_{0}(f) \neq 0$,

(3) the rank of $\pi_{0}\left(f^{x}\right)$ is zero or one for all $x \in G$.

Moreover, there exist $\delta>0$, a compact neighborhood $Z$ of the identity in $G$, and for $x \in Z$ real numbers $\lambda_{x}$ and projections $p_{x}$ in $\mathscr{H}$ (of rank one) with

(4) $\pi_{0}\left(f^{x}\right)=\lambda_{x} p_{x}(x \in Z), \lambda_{x} \geqq \delta$.

Let $Z^{\prime}:=Z P_{0} \leqq X$, let $A_{Z^{\prime}}:=A / k\left(Z^{\prime}\right)$, and let $\psi: A \rightarrow A_{Z^{\prime}}$, be the quotient map. As above, every irreducible representation of $A_{Z^{\prime}}$ is equivalent to one of the form $z \pi_{0}$, $z \in Z$. Let $B$ be the closure of $\psi(f) A_{Z^{\prime}} \psi(f)$ in $A_{Z^{\prime}} \cdot B$ is a symmetric semisimple commutative Banach algebra; the commutativity follows from $\left(z \pi_{0}\right)(B)=\mathbb{C} p_{z}$ for $z \in Z$. Moreover, if we define $\chi_{z}: B \rightarrow \mathbb{C}, z \in Z$, by $\left(z \pi_{0}\right)(b)=\chi_{z}(b) p_{z}$ then $\chi_{z}$ is a multiplicative linear functional. We are going to show that any multiplicative 
linear functional $\chi$ on $B$ is of that form (in fact, the Gelfand space $\hat{B}$ is homeomorphic to $Z^{\prime}$ but we don't use and prove this fact). By the extension property of symmetric algebras (see [13] or [17]) there exists for the given $\chi$ an irreducible involutive representation $\pi$ of $A_{Z}$ and a unit vector $\xi$ in the space of $\pi$ with $\pi(b) \xi=\chi(b) \xi$. Since $\pi$ is equivalent to $z \pi_{0}$ for some $z \in Z$ we may assume that $\pi=z \pi_{0}$. Then $\chi=\chi_{z}$ is an immediate consequence. For the element $\psi(f)^{3} \in B$ we find $\chi_{z}\left(\psi(f)^{3}\right)=\lambda_{z}^{3} \geqq \delta^{3}, z \in Z$. But this implies that $B$ has a unit element $u$ (see e.g. p. 84 in [5]); this element satisfies, of course, the equation $\left(z \pi_{0}\right)(u)=p_{z}$ for all $z \in Z$. Moreover, $\psi(f)^{3}$ is invertible in $B$, there exists an $g \in A$ with $\psi\left(g f^{3}\right)=u$. Finally we choose an $h \in A$ with $\pi_{0}\left(h g f^{3}\right) \neq 0$ and $\hat{h}=0$ outside the neighborhood $Z^{\prime}$ of $P_{0}$. After all. it is easy to verify that the elements $q:=\left(g f^{3}\right)^{*} g f^{3}$ and $p:=q h^{*} h q$ have the indicated properties.

At the end of the proof of the theorem we will use the following.

Lemma 3. Let $V$ be a finite-dimensional real vector space, let $m: V^{2} \rightarrow \mathbb{T}$ be a continuous cocycle, and let $w: V \rightarrow \mathbb{R}$ be a symmetric continuous weight function. i.e. a continuous function with $w(x) \geqq 1, w(x+y) \leqq w(x) w(y)$ and $w(-x)=w(x)$ for all $x, y \in V$. Then the Banach space $L^{1}(V, m, w)=\left\{g: V \rightarrow \mathbb{C} ; g\right.$ measurable, $\left.g w \in L^{1}(V)\right\}$ with the norm $\|g\|_{w}:=\|g w\|_{1}$ becomes an involutive Banach algebra if we define

and

$$
(g * h)(s)=\int_{V} d t \bar{m}(s+t,-t) g(s+t) h(-t)
$$

$$
g^{*}(s)=m(-s, s) \overline{g(-s)} .
$$

If $\alpha$ is an involutive irreducible representation of $L^{1}(V, m, w)$ then the image of $\alpha$ contains a projection of rank one.

Proof. One verifies very easily that $L^{1}(V, m, w)$ is an involutive Banach algebra. Equivalent cocycles give isomorphic algebras. Hence we may assume that $m$ is of the form $m(x, y)=e^{i \mu(x, y)}$ with an antisymmetric bilinear form $\mu$ on $V$. The representation $\alpha$ is obtained by integration from an irreducible $\bar{m}$-projective unitary representation of $V$ which is also denoted by $\alpha$, i.e.

$$
\begin{gathered}
\alpha(g)=\int_{V} d t g(t) \alpha(t), \\
\alpha(x) \alpha(y)=\bar{m}(x, y) \alpha(x+y)
\end{gathered}
$$

for $g \in L^{1}(V, m, w)$ and $x, y \in V$.

By the way, it is clear that the image of the unweighted algebra $L^{1}(V, m)$ under $\alpha$ contains projections of rank one because $L^{1}(V, m)$ is a quotient of the $L^{1}$-algebra of a nilpotent Lie group. In order to show that $\alpha\left(L^{1}(V, m, w)\right)$ contains projections of rank one we will explicitly construct a function $p$ on $V$ (essentially the GauB function) which is integrable against every continuous weight and which is mapped onto a projection of rank one.

Let $K$ be the kernel of the form $\mu$, i.e.

$$
\begin{aligned}
K: & =\{x \in V ; \mu(x, V)=0\}=\{x \in V ; \mu(V, x)=0\} \\
& =\{x \in V ; m(x, V)=1\} .
\end{aligned}
$$


We choose a vector space complement $X$ to $K$ in $V$ and a direct decomposition of $X, X=X_{1}+X_{2}$, such that $\mu\left(X_{j}, X_{j}\right)=0$. Then the restriction of $\mu$ gives a nondegenerate pairing $\mu: X_{1} \times X_{2} \rightarrow \mathbb{R}$. Moreover we choose a positive definite inner product $\langle$,$\rangle on X_{2}, \mu$ and $\langle$,$\rangle give an isomorphism T: X_{1} \rightarrow X_{2}$ defined by $\left\langle T x_{1}, x_{2}\right\rangle=\mu\left(x_{1}, x_{2}\right)$ for $x_{1} \in X_{1}, x_{2} \in X_{2}$. And we get an inner product on $X_{1}$, also denoted by $\langle$,$\rangle , by \langle x, y\rangle:=\langle T x, T y\rangle=\mu(x, T y)$.

For $y \in K$ and $x \in V$ we have

$$
\alpha(x) \alpha(y)=\alpha(x+y)=\alpha(y+x)=\alpha(y) \alpha(x) .
$$

Hence $\left.\alpha\right|_{K}$ is a unitary character of $K$, say $\chi$. By the way, $\alpha$ is uniquely determined by $\chi$. This is well known (the Stone-von Neumann theorem) and can also be deduced from the following considerations. We let the group $X_{1}$ act on $L^{1}\left(X_{2}\right)$ by

$$
f^{x_{1}}\left(x_{2}\right)=m^{2}\left(x_{1}, x_{2}\right) f\left(x_{2}\right) \text { for } x_{1} \in X_{1}, x_{2} \in X_{2} \text {. }
$$

The function $u: X_{2} \rightarrow \mathbb{R}$ is defined by $u(x)=e^{-\langle x, x\rangle}, U$ on $X=X_{1}+X_{2}$ is defined by $U\left(x_{1}+x_{2}\right)=\left(u^{x_{1}} * u\right)\left(x_{2}\right)$ where the star denotes the convolution in $L^{1}\left(X_{2}\right)$ and the function $p$ on $V=X_{1}+X_{2}+K$ is defined by

$$
p\left(x_{1}+x_{2}+k\right)=\bar{m}\left(x_{1}, x_{2}\right)\left(u^{x_{1}} * u\right)\left(x_{2}\right) \varphi(k)=\bar{m}\left(x_{1}, x_{2}\right) U\left(x_{1}+x_{2}\right) \varphi(k)
$$

where $\varphi: K \rightarrow \mathbb{C}$ is any compactly supported continuous function on $K$ with $\int_{K} \varphi(k) \chi(k) d k=1$.

We claim that $p$ is contained in $L^{1}(V, m, w)$ and that $\alpha(p)$ is a projection of rank one (if the measures on $X_{1}$ and $X_{2}$ are suitably normalized). First we compute $U$ more explicitly, this is done in the usual way.

$$
\begin{aligned}
U\left(x_{1}+x_{2}\right) & =\int_{X_{2}} d z u^{x_{1}}(z) u\left(x_{2}-z\right) \\
& =\int_{X_{2}} d z m^{2}\left(x_{1}, z\right) e^{-\langle z, z\rangle-\left\langle x_{2}-z, x_{2}-z\right\rangle} \\
& =\int_{X_{2}} d z m^{2}\left(x_{1}, z\right) e^{-\left\langle\sqrt{2} z-\frac{1}{\sqrt{2}} x_{2}, \sqrt{2} z-\frac{1}{\sqrt{2}} x_{2}\right\rangle-\frac{1}{2}\left\langle x_{2}, x_{2}\right\rangle} \\
& =\frac{1}{\sqrt{2}} m\left(x_{1}, x_{2}\right) e^{-\frac{1}{2}\left\langle x_{2}, x_{2}\right\rangle} \int_{X_{2}} d z m^{2}\left(\frac{x_{1}}{\sqrt{2}}, z\right) u(z)
\end{aligned}
$$

The Fourier transform $\mathscr{F}: L^{1}\left(X_{2}\right) \rightarrow C_{\infty}\left(X_{1}\right)$ is defined by

$$
(\mathscr{F} f)\left(x_{1}\right)=\int_{x_{2}} d x_{2} m^{2}\left(x_{1}, x_{2}\right) f\left(x_{2}\right) .
$$

Putting $v:=\mathscr{F} u$, we get

$$
U\left(x_{1}+x_{2}\right)=\frac{1}{\sqrt{2}} m\left(x_{1}, x_{2}\right) e^{-\frac{1}{2}\left\langle x_{2}, x_{2}\right\rangle} v\left(\frac{x_{1}}{\sqrt{2}}\right) .
$$

In order to compute $v$ we fix a nonzero $x$ in $X_{1}$ and form the function $\psi: \mathbb{R} \rightarrow \mathbb{C}$, $\psi(t)=v(t x)$. Let $Y_{2}:=\left\{y \in X_{2} ; \mu(x, y)=0\right\}$ and let $w:=T x \in X_{2}$. Then $Y_{2}+\mathbb{R} w$ is an 
orthogonal decomposition of $X_{2}$ (with respect to $\langle$,$\rangle ). If we normalize the$ Lebesgue measure on $Y_{2}$ suitably we get

if we put $E=\int_{Y_{2}} d y u(y)$.

$$
\begin{aligned}
\psi(t) & =v(t x)=\int_{Y_{2}} d y \int_{-\infty}^{\infty} d s m^{2}(t x, y+s w) u(y+s w) \\
& =E \int_{-\infty}^{\infty} d s m^{2}(t x, s w) u(s w)
\end{aligned}
$$

For the derivative of $\psi$ we find

$$
\psi^{\prime}(t)=E i \mu(x, w) \int_{-\infty}^{\infty} d s 2 s m^{2}(t x, s w) u(s w)
$$

or, using integration by parts,

But

$$
\begin{aligned}
\psi^{\prime}(t) & =-2 t E \frac{\mu(x, w)^{2}}{\langle w, w\rangle} \int_{-\infty}^{\infty} d s m^{2}(t x, s w) u(s w) \\
& =-2 t \frac{\mu(x, w)^{2}}{\langle w, w\rangle} \psi(t) .
\end{aligned}
$$

$$
\mu(x, w)=\mu(x, T x)=\langle x, x\rangle, \quad\langle w, w\rangle=\langle T x, T x\rangle=\langle x, x\rangle
$$

and hence

$$
\psi^{\prime}(t)=-2 t\langle x, x\rangle \psi(t) .
$$

From this differential equation it follows that there is a constant $C$ with

$$
\psi(t)=C e^{-\langle x, x\rangle t^{2}}=v(t x) .
$$

Putting $t=0$ we see that this constant is independent of $x$ (and positive), putting $t=1$ we find

$$
v(x)=(\mathscr{F} u)(x)=C e^{-\langle x, x\rangle}
$$

and hence

with $C^{\prime}=\frac{C}{\sqrt{2}}$.

$$
U\left(x_{1}+x_{2}\right)=C^{\prime} m\left(x_{1}, x_{2}\right) e^{-\frac{1}{2}\left\langle x_{2}, x_{2}\right\rangle} e^{-\frac{1}{2}\left\langle x_{1}, x_{1}\right\rangle}
$$

Of course, we might use this equation as the definition of $U$, but then it would be more difficult to show that $\alpha(p)$ is a projection of rank one.

Now it is very easy to see that $p$ is contained in $L^{1}(V, m, w)$. Since $w$ is a continuous weight function, for every norm \|\| on $V$ there exists a constant $D>0$ with $w(x) \leqq D e^{D\|x\|}$ for all $x \in V$. Especially we find an $D>0$ with

Then we get

$$
w(x) \leqq D e^{D\langle x, x\rangle\rangle^{1 / 2}} \quad \text { for } x \text { in } X_{1} \text { or in } X_{2} .
$$

$$
\begin{aligned}
& \int_{V} d x|p(x)| w(x) \leqq \int_{X_{1}} d x_{1} \int_{X_{2}} d x_{2} \int_{K} d k|\varphi(k)|\left|U\left(x_{1}+x_{2}\right)\right| w\left(x_{1}\right) w\left(x_{2}\right) w(k) \\
& \leqq C^{\prime} D^{2} \int_{X_{1}} d x_{1} \int_{X_{2}} d x_{2} \int_{K} d k w(k)|\varphi(k)| e^{-\frac{1}{2}\left\langle x_{2}, x_{2}\right\rangle} \\
& \cdot e^{-\frac{1}{2}\left\langle x_{1}, x_{1}\right\rangle} e^{D\left\langle x_{1}, x_{1}\right\rangle^{1 / 2}} e^{D\left\langle x_{2}, x_{2}\right\rangle^{1 / 2}}<\infty
\end{aligned}
$$


In order to show that $\alpha(p)$ is a projection of rank one we observe that the projective representation $\alpha$ of $V$ gives an irreducible involutive representation of the unweighted algebra $L^{1}(X, m)$, also denoted by $\alpha$. The latter algebra is *-isomorphic to the Leptin algebra $L^{1}\left(X_{1}, L^{1}\left(X_{2}\right), T\right)$ with multiplication

$$
(g * h)(x)=\int_{X_{1}} d y g(x+y)^{-y} * h(-y)
$$

and involution $g^{*}(x)=g(-x)^{x *}$ where the action of $X_{1}$ on $L^{1}\left(X_{2}\right)$ is as above, i.e. $f^{x}(y)=m^{2}(x, y) f(y), x \in X_{1}, y \in X_{2}$. In fact, the map $g \rightarrow g^{\prime}, g^{\prime}\left(x_{1}\right)\left(x_{2}\right)=m\left(x_{1}, x_{2}\right) g\left(x_{1}\right.$ $\left.+x_{2}\right)$, from $L^{1}(X, m)$ onto $L^{1}\left(X_{1}, L^{1}\left(X_{2}\right), T\right)$ is an isomorphism. The irreducible involutive representation $\alpha^{\prime}$ of $L^{1}\left(X_{1}, L^{1}\left(X_{2}\right), T\right)$ is defined by $\alpha^{\prime}\left(g^{\prime}\right)=\alpha(g)$ for $g \in L^{1}(X, m)$.

With these notations we have

$$
\begin{aligned}
\alpha(p) & =\int_{X_{1}} d x_{1} \int_{X_{2}} d x_{2} \int_{K} d k \bar{m}\left(x_{1}, x_{2}\right) U\left(x_{1}+x_{2}\right) \varphi(k) \alpha\left(x_{1}+x_{2}\right) \chi(k) \\
& =\int_{X_{1}} d x_{1} \int_{X_{2}} d x_{2} \bar{m}\left(x_{1}, x_{2}\right) U\left(x_{1}+x_{2}\right) \alpha\left(x_{1}+x_{2}\right)=\alpha^{\prime}(U) .
\end{aligned}
$$

Recall that $U: X_{1} \rightarrow L^{1}\left(X_{2}\right)$ is given by $U x=u^{x} * u . U$ is an hermitean element and one computes very easily (by applying $\mathscr{F}$ ) that

$$
U * U=U \int_{X_{1}}(\mathscr{F} u)(y)^{2} d y,
$$

hence $U * U=U$ if the Lebesgue measure on $X_{1}$ is suitably normalized, and that

$$
U * L^{1}\left(X_{1}, L^{1}\left(X_{2}\right), T\right) * U=\mathbb{C} U .
$$

From these facts and the irreducibility of $\alpha^{\prime}$ it follows that $\alpha^{\prime}(U)=\alpha(p)$ is a projection of rank one or zero. But it cannot be zero because the algebra $L^{1}\left(X_{1}, L^{1}\left(X_{2}\right), T\right)$ is simple, see [12], Theorem 4.

Proof of the Theorem. The unitary representation theory of exponential Lie groups is very well understood. The set $\hat{G}$ of equivalence classes of irreducible unitary representations of the exponential Lie group $G$ is in a canonical bijective correspondence with the set of $G$-orbits (under the coadjoint action) in $\mathfrak{g}^{*}$, the real dual of the Lie algebra $g$ of $G$, see e.g. [12]. In the sequel, $I$ will describe (and use) a somewhat unusual procedure to obtain all the irreducible unitary representations of $G$.

Let $N$ be the nilradical of $G$ and denote by $\varphi: G \rightarrow G / N$ the quotient morphism. We start with an irreducible unitary representation $\tau$ of $N$. Let $G_{\tau}$ be the stabilizer of (the class of) $\tau$ in $\hat{N}, G_{\tau}$ is known to be a connected group. We choose a complement $W$ to $G_{v} / N$ in the vector group $G / N, G / N=G_{z} / N \times W$, and denote by $H$ the preimage of $W$ under $\varphi$. Then we induce $\tau$ up to $H, \gamma: \underset{N}{\operatorname{H}} \boldsymbol{H} \tau . \gamma$ is an irreducible unitary representation of $H$ as $H_{\tau}=G_{\tau} \cap H=N$. Moreover the class of $\gamma$ in $\hat{H}$ is invariant under $G$. Therefore, $\gamma$ extends to a projective unitary representation $\tilde{\gamma}$ of $G$. To be more specific, $\tilde{\gamma}(x) \tilde{\gamma}(y)=\tilde{\gamma}(x y) m(x, y)$ for $x, y \in G$ with a continuous 2-cocycle $m$ living on $G / H$. Then let $x$ be an irreducible $\bar{m}$-projective representation of $G / H$ and form the tensor product $\tilde{\gamma} \otimes x$, which is of course an 
ordinary (irreducible unitary) representation of $G$. By varying $\tau$ and $x$ every irreducible unitary representation of $G$ is obtained in that way. But now we consider $\tau, W, \gamma, \tilde{\gamma}, m$ and $x$ as fixed and let $\pi:=\tilde{\gamma} \otimes x$. We have to show that $\pi\left(L^{1}(G)\right)$ contains a projection of rank one. In the following main step we show that $\gamma\left(L^{1}(H)\right)$ contains a projection of rank one.

It is well known that the $G$-orbits in $\hat{N}$ are locally closed and by the theorem of Brown, $\hat{N}$ is homeomorphic to the space of $N$-orbits in $n^{*}$. Since $L^{1}(N)$ is *-regular, also the $G$-orbits in $\operatorname{Priv}_{*}\left(L^{1}(N)\right)$ are locally closed. Let

$$
X:=\left\{\operatorname{ker} \tau^{x} ; x \in G\right\} \subseteq \operatorname{Priv}_{*}\left(L^{1}(N)\right),
$$

let $Y:=\bar{X} \backslash X$ and let $I_{1}$ and $I_{2}$ be the kernels of the closed sets $Y$ and $\bar{X}$, respectively. Of course, $I_{1}$ and $I_{2}$ are $G$-invariant ideals in $L^{1}(N)$. We note that the quotient $I:=I_{1} / I_{2}$ satisfies the assumptions of Lemma 2 . It is symmetric because $L^{1}(N)$ is symmetric by [14], and $\operatorname{Priv}_{*}(I)$ is homeomorphic to $X$. Now, using a (continuous) cross section $\sigma: W \rightarrow H$ (with $\sigma(0)=e$ and $\sigma(-x)=\sigma(x)^{-1}$ ) to $\varphi: H \rightarrow H / N=W$ we write the algebra $L^{1}(H)$ as $L^{1}\left(W, L^{1}(N), P, T\right)$ in the sense of [10]. To be more specific: If $\delta$ is the modular function of the action of $G$ on $N$, i.e. $d\left(x n x^{-1}\right)=\delta(x)^{-1} d n(n \in N, x \in G)$ we define an action of $G$ on $L^{1}(N)$ by

$$
a^{x}(n)=\delta(x)^{-1} a\left(x n x^{-1}\right) .
$$

The (not necessarily homomorphic) map $T: W \rightarrow \operatorname{Aut}\left(L^{1}(N)\right)$ is defined by $T_{\mathrm{c}} a$ $=a^{\sigma(-s)}$. For $s, t \in W$ the multiplier $P_{s, t}: L^{1}(N) \rightarrow L^{1}(N)$ is defined by

$$
\left(P_{s, t} a\right)(n):=a\left(\sigma(t)^{-1} \sigma(s)^{-1} \sigma(s+t) n\right) .
$$

Then the convolution in $L^{1}\left(W, L^{1}(N), P, T\right)$ is given by

$$
(f * g)(s)=\int_{W} d t\left[P_{s+t,-t} T_{\sigma(t)} f(s+t)\right] g(-t),
$$

and the involution is given by $f^{*}(s)=f(-s)^{* \sigma(s)}$.

Since (the restriction of the representation $\gamma$ vanishes on $I_{2}$, we may consider it as a representation of $L^{1}\left(W, L^{1}(N) / I_{2}, P, T\right)$ with induced action $T$ and an induced family of multipliers $P_{s, t}$ or as a representation of $L^{1}(W, I, P, T)$. Since $\gamma$ is up to equivalence the unique irreducible representation of $L^{1}(W, I, P, T)$ the image of $L^{1}(W, I, P, T)$ under $\gamma$ consists of compact operators. Next we choose $p, q \in I$ as in Lemma 2 and let $J$ be the smallest closed $G$-invariant ideal in $I$ containing $p$, i.e. $J=\left[I\left\{p^{t} ; t \in G\right\} I\right]^{-}$. We want to apply Lemma 1 to the algebra $L^{1}(W, J, P, T)$ in order to get finite rank operators in the image of $\gamma$. Since $L^{1}(W, J, P, T)$ has only one irreducible representation (namely the restriction of $\gamma$ ) it remains to show that $L^{1}(W, J, P, T)$ is symmetric. To this end, we choose a lattice $W_{d}$ in $W$ such that the supports of the "Fourier transforms" $\hat{q}$ and $\left(q^{\sigma(s)}\right), \hat{q}: X \rightarrow \bigcup_{P \in X} l / P$, are disjoint for all nonzero $s$ in $W_{d}$. This is possible because $X$ is a simply transitive $W$-space. By the way, we might have chosen the lattice first and then ( $p$ and) $q$ such that the above property is satisfied.

Next, $I$ will show that it suffices to prove the symmetry of $L^{1}\left(W_{d}, J, P, T\right)$ (with restricted action and multipliers) by applying Satz 2 in [16] finitely often. Let 
$w_{1}, \ldots, w_{d}$ be a $\mathbb{Z}$-basis of $W_{d}$, let

$$
W_{k}:=L_{\mathbb{Z}}\left(w_{1}, \ldots, w_{k}\right)+L_{\mathbb{R}}\left(w_{k+1}, \ldots, w_{d}\right) \text { for } 0 \leqq k \leqq d,
$$

and let

$$
U_{k}:=L_{\mathbb{Z}}\left(w_{1}, \ldots, w_{k-1}\right)+L_{\mathbb{R}}\left(w_{k+1}, \ldots, w_{d}\right) \text { for } 1 \leqq k \leqq d .
$$

Then we have $W_{k}=U_{k}+\mathbb{Z} w_{k}$ and $W_{k-1}=U_{k}+\mathbb{R} x_{k}$ for $1 \leqq k \leqq d$. The algebra $L^{1}\left(W_{k-1}, J, P, T\right)$ is isomorphic to a certain subquotient of the $L^{1}$-algebra of $\varphi^{-1}\left(W_{k-1}\right)$. Since the group $\varphi^{-1}\left(W_{k-1}\right)$ is isomorphic to a semidirect product of $\mathbb{R}$ and $\varphi^{-1}\left(U_{k}\right)$, it follows that $L^{1}\left(W_{k-1}, J, P, T\right)$ is isomorphic to $L^{1}\left(\mathbb{R}, Q_{k}, T_{k}\right)$ where $Q_{k}$ is a certain subquotient of $L^{1}\left(\varphi^{-1}\left(U_{k}\right)\right), T_{k}$ is an (homomorphic!) action of $\mathbb{R}$ on $Q_{k}$. and there is no factor system. Moreover, $L^{1}\left(W_{k}, J, P, T\right)$ is isomorphic to $L^{1}\left(\mathbb{Z}, Q_{k}, T_{k}\right)$. Satz 2 in [16] tells us that for $1 \leqq k \leqq d$ the symmetry of $L^{1}\left(W_{k}, J, P, T\right)$ implies the symmetry of $L^{1}\left(W_{k-1}, J, P, T\right)$. Starting with $k=d$ we conclude that $L^{1}\left(W_{0}=W, J, P, T\right)$ is symmetric.

In order to prove the symmetry of $L^{1}\left(W_{d}, J, P, T\right)$ we take a simple module $E$ over this algebra and show that there exists an involutive representation of $L^{1}\left(W_{d}, J, P, T\right)$ in an Hilbert space $\mathscr{H}$ and an embedding of $E$ in $\mathscr{H}$ which is linear over $L^{1}\left(W_{d}, J, P, T\right)$. $E$ may also be considered as a (simple) module over $L:=L^{1}\left(W_{d}, I, P, T\right)$. Moreover, $I$ is contained in $L$. From the definition of $J$ it follows that there exists $t \in G$ with $p^{t} E \neq 0$. W.l.o.g. we may assume that $t=e$. The following properties are important for the rest of the proof:

(1) $q * L * q=q I q \subseteq I$.

(2) $q I q$ is a commutative subalgebra of $I$; in particular, $p$ commutes with $q I q$.

(3) $q E$ is onedimensional.

$\operatorname{ad}(1)$ : Let $f \in L$. Then one computes $(q * f * q)(s)=q^{\sigma(s)} f(s) q$ for $s \in W_{d}$. Since for $s \neq 0$ the supports of $\hat{q}$ and $\left(q^{\sigma(s)}\right)^{\wedge}$ are disjoint and since $I$ is semisimple we find that $(q * f * q)(s)=0$ for $s \neq 0$. This shows that $q * f * q=q * f(0) * q \in q I q \leqq I$

(2) is obvious.

$\operatorname{ad}(3)$ : Let $\eta$ be any nonzero element in $p E$. From $q p=p$ it follows that $q \eta=\eta$. Since $E$ is simple we have $E=L \eta=L q \eta$ and hence $q E=q L q \eta=q I q \eta$. From the fact that $p$ commutes with $q I q$ we get $q I q \eta \subseteq q I q p E=p q I q E \subseteq p E$, and, therefore, $q E$ $=q I q \eta \subseteq p E$ for all nonzero $\eta$ in $p E$. Together with $p E \subseteq\{\xi \in E ; q \xi=\xi\} \subseteq q E$ it follows that $p E=q E$ is a closed subspace of $E$. From all this we deduce that $q E$ is a simple module over the commutative Banach algebra $(q I q)^{-}$and hence onedimensional.

The $I$-submodule $I q E$ of $E$ is cyclic, consequently it possesses a simple quotient. To this simple quotient there exists (because $I$ is symmetric) an irreducible involutive representation $\varrho$ of $I$ (i.e. an element in the $G$-orbit of $\tau$ ) in an Hilbert space $\mathscr{K}$ and an $I$-linear embedding of the quotient into $\mathscr{K}$. By composition, we get a nonzero $I$-linear map $I q E \rightarrow \mathscr{K} . \varrho$ may be considered as an involutive representation of $L^{1}(N)$ and as an irreducible unitary representation of $N$. We form the induced representation $\varrho^{\prime}={ }_{N} \boldsymbol{H}_{d}$ ind $\varrho$ with $H_{d}:=\varphi^{-1}\left(W_{d}\right)$ in the space $\mathscr{H}$ and claim that there exists an $L$-linear embedding of $E$ in $\mathscr{H}$ (the representation $\varrho^{\prime}$ of $L^{1}\left(H_{d}\right)$ may be considered as an representation of $\left.L=L^{1}\left(W_{d}, I, P, T\right)\right)$ which is a subquotient of $L^{1}\left(H_{d}\right)$. By construction, $\varrho(q)$ is an operator of rank $\leqq 1$. Since there is a nonzero $I$-linear map $I q E \rightarrow \mathscr{K}$ and since the elements of $q E$ are fixed by 
$q, \varrho(q)$ has to be a projection of rank one. The condition on the support of $\hat{q}$ implies that $\varrho\left(q^{x}\right)=0$ for all $x \in H_{d} \backslash N$. Consequently, also $\varrho^{\prime}(q)$ is a projection of rank one. Moreover, the onedimensional $q I q$-module $\varrho^{\prime}(q) \mathscr{H}$ is isomorphic to $\varrho(q) \mathscr{K}$ and hence to the $q I q$-module $q E$. Let $\eta$ and $\eta^{\prime}$ be nonzero elements in $q E$ and $\varrho^{\prime}(q) \mathscr{H}$, respectively. Then we define $S: E \rightarrow \mathscr{H}$ by $S(f \eta)=\varrho^{\prime}(f) \eta^{\prime}$ for $f \in L$. $S$ is well-defined. We have to show that $f \eta=0$ implies $\varrho^{\prime}(f) \eta^{\prime}=0$. From $f \eta=0$ and $q \eta=\eta$ we obtain $q f^{*} g q \eta=0$ and hence $\varrho^{\prime}\left(q f^{*} f q\right) \eta^{\prime}=0$ because $q E$ and $\varrho^{\prime}(q) \mathscr{H}$ are isomorphic $q I q$ $=q L q$-modules. But $\varrho^{\prime}\left(q f^{*} f q\right) \eta^{\prime}=0$ implies $\left\langle\varrho^{\prime}(f q) \eta^{\prime}, \varrho^{\prime}(f q) \eta^{\prime}\right\rangle=0$ and hence

$$
0=\varrho^{\prime}(f q) \eta^{\prime}=\varrho^{\prime}(f) \varrho^{\prime}(q) \eta^{\prime}=\varrho^{\prime}(f) \eta^{\prime} .
$$

Obviously, $S$ is an $L$-linear embedding. Now the symmetry of $L^{1}\left(W_{d}, J, P, T\right)$ [and hence of $\left.L^{1}(W, J, P, T)\right]$ is proved, and Lemma 1 gives the existence of rank one projections in

$$
\gamma\left(L^{1}(W, J, P, T)\right) \subseteq \gamma\left(L^{1}(H)\right) .
$$

Let $A:=L^{1}(H) / \operatorname{ker} \gamma$. Since $\operatorname{ker} \gamma$ is invariant under $G$ the group $G$ acts on $A$. We consider $\gamma$ as a representation of $A$ and choose an $a \in A$ such that $\gamma(a)$ is a projection of rank one, say $\gamma(a)=\langle-, \xi\rangle \xi$ with some unit vector $\xi$ in the representation space $\mathscr{H}_{\gamma}$ of $\gamma$. For every $x \in G$ the space $a^{x} A a$ is onedimensional. In the following, we will pick up a particular basis vector:

(4) There is a unique continuous function $f: G \rightarrow A$ with $\gamma(f(x))=\langle-, \xi\rangle$ $\tilde{\gamma}(x)^{-1} \xi . f(x)$ is contained in $a^{x} A a$. The function $w: G \rightarrow \mathbb{R}$, defined by $w(x)=\|f(x)\|$, is constant on cosets modulo $H$. Moreover, $w\left(x^{-1}\right)=w(x)$ and $w(x) \geqq 1$ for all $x \in G$.

$\operatorname{ad}(4)$ : The uniqueness of $f$ is obvious. For $x \in G$ we have $\gamma\left(a^{x}\right)=\tilde{\gamma}(x)^{-1} \gamma(a) \tilde{\gamma}(x)$ and hence, for $b \in A, \gamma\left(\mathrm{a}^{x} \mathrm{ba}\right)=\langle-, \xi\rangle\langle\tilde{\gamma}(x) \gamma(b) \xi, \xi\rangle \tilde{\gamma}(x)^{-1} \xi$. Let $x \in G$ be given. We choose an $b \in A$ with $\langle\tilde{\gamma}(x) \gamma(b) \xi, \xi\rangle \neq 0$; this is possible because $\gamma$ is irreducible. Then we define $f(y)=a^{y} b a\langle\tilde{\gamma}(y) \gamma(b) \xi, \xi\rangle^{-1}$ for $y$ in a suitable neighborhood of $x$. The group $H$ acts by (left) translations isometrically on $L^{1}(H)$ and also on $A$. One computes very easily that $f(x h) \in A(x \in G, h \in H)$ is nothing else but $f(x)$ translated by $h$ and, consequently, $\|f(x h)\|=\|f(x)\|$. From $\|\gamma(f(x))\|=1$ it follows that $w(x) \geqq 1$, the equation $w\left(x^{-1}\right)=w(x)$ is a consequence of $\|f(x)\|=\left\|f\left(x^{-1}\right)^{* x}\right\|$ $=\left\|f\left(x^{-1}\right)\right\|$.

Next, we choose a continuous cross section $\sigma: G / H \rightarrow G$ with $\sigma(e)=e$ and $\sigma\left(x^{-1}\right)$ $=\sigma(x)^{-1}$ (the original $\sigma: V \rightarrow H$ is not longer used) and identify $L^{1}(G)$ with $L^{1}\left(G / H, L^{1}(H), P, T\right)$ in a similar way as we did for $L^{1}(H)$. From now, we write $V$ for the vector group $G / H$. The ideal ker $\gamma$ is stable under $P$ and $T$. Hence we may form $B:=L^{1}(V, A, P, T)$ which is a quotient of $L^{1}(G)$. We note that $\pi=\tilde{\gamma} \otimes \alpha$ (considered as representation of $L^{1}(G)$ ), factorizes through $L^{1}(G) \rightarrow B$. Moreover, $A$ is (in a canonical way) contained in the multiplier algebra of $B$, we have $(c * g)(t)$ $=c^{\sigma(t)} g(t)$ and $(g * c)(t)=g(t) c$ for $t \in V, c \in A$ and $g \in B$. We wish to compute the (involutive Banach) subalgebra $a * B * a$ of $B$. Let $g=a * g * a$ be a element of $a * B * a$. Then the equation $g(t)=a^{\sigma(t)} g(t) a$ holds for almost all $t \in V$, i.e. $g(t)$ is (a.e.) a scalar multiple of $f(\sigma(t))$. From this observation one obtains very easily the following fact:

(5) For $h \in L^{1}(V, w):=\left\{h: V \rightarrow \mathbb{C} ; h\right.$ measurable, $\left.h w \in L^{1}(V)\right\}$ we define $h^{\prime}: V \rightarrow A$ by $h^{\prime}(t)=h(t) f(\sigma(t))$. Then $h \rightarrow h^{\prime}$ is an isometry from the Banach space $L^{1}(V, w)$ onto $a * B * a$. 
By "transport de la structure" $L^{1}(V, w)$ is considered as an involutive Banach algebra. For the multiplication (also written as *) and the involution in $L^{1}(V, w)$ one finds

$$
\begin{aligned}
(g * h)(s) & =\int_{V} d t \bar{m}(s+t,-t) g(s+t) h(-t), \\
g^{*}(s) & =m(-s, s) \overline{g(-s)}
\end{aligned}
$$

Note that the cocycle $m$ on $G$ may be considered as a function on $V^{2}$.

ad (6): By definition, we have

And

$$
\begin{aligned}
\left(g^{\prime} * h^{\prime}\right)(s) & =\int_{V} d t\left[P_{s+t,-i} T_{\sigma(t)} g^{\prime}(s+t)\right] h^{\prime}(-t) \\
& =\int_{V} d t g(s+t) h(-t)\left[P_{s+t,-t} T_{\sigma(t)} f(\sigma(s+t))\right] f(\sigma(-t)) .
\end{aligned}
$$

$$
\begin{aligned}
& \gamma\left(\left[P_{s+t,-t} T_{\sigma(t)} f(\sigma(s+t))\right] f(\sigma(-t))\right) \\
&= \gamma\left(\sigma(s)^{-1} \sigma(s+t) \sigma(t)^{-1}\right) \gamma\left(f(\sigma(s+t))^{\sigma(-t)}\right) \gamma(f(\sigma(-t)) \\
&= \gamma\left(\sigma(s)^{-1} \sigma(s+t) \sigma(t)^{-1}\right) \tilde{\gamma}(\sigma(-t))^{-1} \\
& \circ\langle-, \xi\rangle \tilde{\gamma}(\sigma(s+t))^{-1} \xi \circ \tilde{\gamma}(\sigma(-t)) \circ\langle-, \xi\rangle \tilde{\gamma}(\sigma(-t))^{-1} \xi \\
&=\langle-, \xi\rangle \gamma\left(\sigma(\mathrm{s})^{-1} \sigma(\mathrm{s}+\mathrm{t}) \sigma(\mathrm{t})^{-1}\right) \tilde{\gamma}(\sigma(-\mathrm{t}))^{-1} \tilde{\gamma}(\sigma(\mathrm{s}+\mathrm{t}))^{-1} \xi \\
&=\langle-, \xi\rangle\left\{\tilde{\gamma}(\sigma(\mathrm{s}+\mathrm{t})) \tilde{\gamma}(\sigma(-\mathrm{t})) \gamma\left(\sigma(\mathrm{t}) \sigma(\mathrm{s}+\mathrm{t})^{-1} \sigma(\mathrm{s})\right\}^{-1} \xi\right. \\
&=\langle-, \xi\rangle\left\{\tilde{\gamma}\left(\sigma(\mathrm{s}+\mathrm{t}) \tilde{\gamma}\left(\sigma(\mathrm{s}+\mathrm{t})^{-1} \sigma(\mathrm{s})\right)\right\}^{-1} \xi\right. \\
&=\langle-, \xi\rangle\{\mathrm{m}(\mathrm{s}+\mathrm{t},-\mathrm{t}) \tilde{\gamma}(\sigma(\mathrm{s}))\}^{-1} \xi=\overline{\mathrm{m}}(\mathrm{s}+\mathrm{t},-\mathrm{t}) \gamma(\mathrm{f}(\sigma(\mathrm{s}))) .
\end{aligned}
$$

Consequently,

$$
\left(g^{\prime} * h^{\prime}\right)(s)=\int_{V} d t g(s+t) h(-t) \bar{m}(s+t, t) f(\sigma(s))=(g * h)^{\prime}(s) .
$$

The formula for the involution follows from the equation $f\left(x^{-1}\right)^{* x}$ $=m\left(x^{-1}, x\right) f(x)$ for $x \in G$.

From the facts that $w$ is continuous and that $L^{1}(V, w)$ is a Banach algebra one deduces very easily that $w$ is a weight function on $V$, i.e. $L^{1}(V, w)$ is an algebra of the type $L^{1}(V, m, w)$ as considered in Lemma 3. $\pi(a * B * a)$ annihilates the orthogonal complement of $\pi(a) \mathscr{H}_{\pi}$ in $\mathscr{H}_{\pi}$, and $\pi$ gives by restriction an irreducible involutive representation of $a * B * a$ in $\pi(a) \mathscr{H}_{n}$ which is by the way canonically isomorphic to the representation space of $\alpha$. Since under the identification of $a * B * a$ with $L^{1}(V, m, w)$ we may consider $\pi(a) \mathscr{H}_{n}$ as an irreducible $L^{1}(V, m, w)$-module, Lemma 3 shows that there is an $g \in L^{1}(V, m, w)$ such that $\pi\left(g^{\prime}\right)$ is a projector of rank one in $\pi(a) \mathscr{H}_{\pi}$ and then also a projector of rank one in $\mathscr{H}$ which proves the theorem.

We conclude this article with an application of the theorem.

Remark. Let $G$ be an exponential Lie group. If $L^{1}(G)$ is symmetric then $L^{1}(G)$ is *-regular.

This remark was already proved in [15] under an additional hypothesis which was only needed in order to establish the existence of finite rank operators in the image of irreducible unitary representations. The converse of the remark is still open, a partial result is contained in the same paper. 


\section{References}

1. Arsac, G.: Opérateurs compacts dans l'espace d'une représentation, preprint

2. Bernat, P.: Représentations des groupes de Lie résolubles. Paris: Dunod 1972

3. Boidol, J.: *-regularity of exponential Lie groups, Invent. Math. 56, 231-238 (1980)

4. Boidol, J.: Räume primitiver Ideale von Gruppenalgebren. Math. Ann. 236, 1-13 (1978)

5. Bonsall, F.F., Duncan, I.: Complete normed algebras. Ergebnisse der Mathematik 80. Berlin, Heidelberg, New York: Springer 1973

6. Brown, 1.: Dual topology of a nilpotent Lie group. Ann. Sci. École Norm. Sup. 6, 407-411 (1973)

7. Charbonnel, J.-Y.: Sur les semi-caractères des groupes de Lie résolubles connexes. J. Functional Analysis 41, 175-203 (1981)

8. Dixmier, J.: Opérateurs de rang fini dans les représentations unitaires. Publ. math. Inst. Hautes Études Sci. 6, 305-317 (1960)

9. Guichardet, A. : Caractères des algèbres de Banach involutives. Ann. Inst. Fourier 13, 1-81 (1963)

10. Leinert, M.: Beitrag zur Theorie der verallgemeinerten $L^{1}$-Algebren. Arch. Math. 21, 594-600 (1970)

11. Leptin, H.: Symmetrie in Banachschen Algebren. Arch. Math. 27, 394-400 (1976)

12. Leptin, H., Poguntke, D.: Symmetry and nonsymmetry for locally compact groups. J. Functional Analysis 33, 119-134 (1979)

13. Naimark, M.A.: Normed algebras, $3^{\text {rd }}$ edition. Groningen: Wolters and Noordhoff 1972

14. Poguntke, D.: Nilpotente Liesche Gruppen haben symmetrische Gruppenalgebren. Math. Ann. 227, 51-59 (1977)

15. Poguntke, D.: Symmetry and nonsymmetry for a class of exponential Lie groups. J. Reine Angew. Math. 315, 127-138 (1980)

16. Poguntke, D.: Einfache Moduln über gewissen Banachschen Algebren: ein Imprimitivitätssatz. Math. Ann. 259, 245-258 (1982)

17. Rickart, C.E.: General theory of Banach algebras. New York : Van Nostrand 1960

Received October 7, 1981 